\title{
Experience With Nifekalant Hydrochloride in a Patient With Ischemic Cardiomyopathy and Severe Ventricular Dysfunction After Dor Operation
}

\author{
Kiyokazu KoKAJI, ${ }^{1} \mathrm{MD}$, Masahiko OKAмото,${ }^{1} \mathrm{MD}$, Kentaro HotodA, ${ }^{1} \mathrm{MD}$, \\ and Hiroya KUMAMARU, ${ }^{2} \mathrm{MD}$
}

\begin{abstract}
SUMMARY
A 52-year-old male with ischemic cardiomyopathy and severe ventricular dysfunction underwent coronary artery bypass grafting and left ventricular reconstruction (Dor operation). The patient developed acute onset of incessant ventricular tachycardia in the early postoperative period that was refractory to therapy with class I antiarrhythmic agents, and multiple attempts at electrical cardioversion were required. A combination of intravenous nifekalant hydrochloride and enteral amiodarone was elected as treatment for this recurrent incessant ventricular tachycardia. Nifekalant hydrochloride was administered as a loading dose $(0.3 \mathrm{mg} / \mathrm{kg} / 5 \mathrm{~min})$, followed by an intravenous infusion $(0.4 \mathrm{mg} / \mathrm{kg} / \mathrm{hr})$. Several days after initiating therapy, the patient no longer experienced episodes of ventricular tachycardia, and there was no compromise in hemodynamics. We conclude that nifekalant hydrochloride is a useful agent for suppression of ventricular tachycardia in patients with severe left ventricular dysfunction, especially during the early postoperative period. (Jpn Heart J 2004; 45: 691-695)
\end{abstract}

Key words: Nifekalant hydrochloride, Dor operation, Ischemic cardiomyopathy, Incessant ventricular tachycardia

VENTRICULAR tachycardia (VT) is a potentially fatal complication that can occur after a Dor operation when employed for patients with ischemic cardiomyopathy and severe left ventricular dysfunction. We describe a case of incessant VT after Dor operation that was successfully treated with nifekalant hydrochloride (NIF).

\section{CASE REPORT}

A 52-year-old male with orthopnea was diagnosed with severe heart failure (NYHA class IV) and was admitted to our hospital for further evaluation and

From the ${ }^{1}$ Department of Cardiovascular Surgery, Kawasaki Municipal Hospital, Kawasaki, and ${ }^{2}$ Department of Surgery, Keio University, Tokyo, Japan.

Address for correspondence: Kiyokazu Kokaji, MD, Department of Surgery, Keio University, 35 Shinanomachi, Shinjuku-

ku, Tokyo 160-8582, Japan.

Received for publication July 30, 2003.

Revised and accepted January 29, 2004. 
treatment. On coronary angiography, total occlusion of native coronary arteries was found in segments 2, 7 and 13, and coronary blood flow was maintained only by collateral circulation from the obtuse marginal branch (segment 12). Furthermore, $75 \%$ stenosis was observed proximally in segment 12 . Left cardiac catheterization revealed prominent left ventricular (LV) dilatation associated with severe LV dysfunction (LVEDVI $181 \mathrm{~mL} / \mathrm{m}^{2}$, LVESVI $142 \mathrm{~mL} / \mathrm{m}^{2}$, LVEF 18\%). A diagnosis of ischemic cardiomyopathy with severe LV dysfunction was made.

The clinical course of the patient is illustrated in the Figure. The patient underwent a Dor operation with concomitant coronary artery bypass grafting. The basic technique was essentially identical to that described by Dor ${ }^{1,2)}$ and performed during total cardiac arrest with cold crystalloid cardioplegia, topical cooling, and terminal warm blood cardioplegia. Bypass grafting to the obtuse marginal branch, posterolateral branch (sequential bypass) and segment 3 of the right coronary artery was performed with saphenous vein grafts, and left anterior descending artery (LAD) bypass grafting was performed with the left internal thoracic artery. Next, the LV was opened between the LAD and the diagonal branch, in the region of the anterior akinetic zone. The infarcted area covered nearly the entire septal and free wall between the apex and both papillary muscle roots. The endocardial scar was mobilized and resected along half of the septal long axis and to the level of both papillary muscle roots on the free wall side. Cryotherapy was then employed along the edge of resection at the transitional zone between the scarred and normal tissue. As the endoventricular purse string

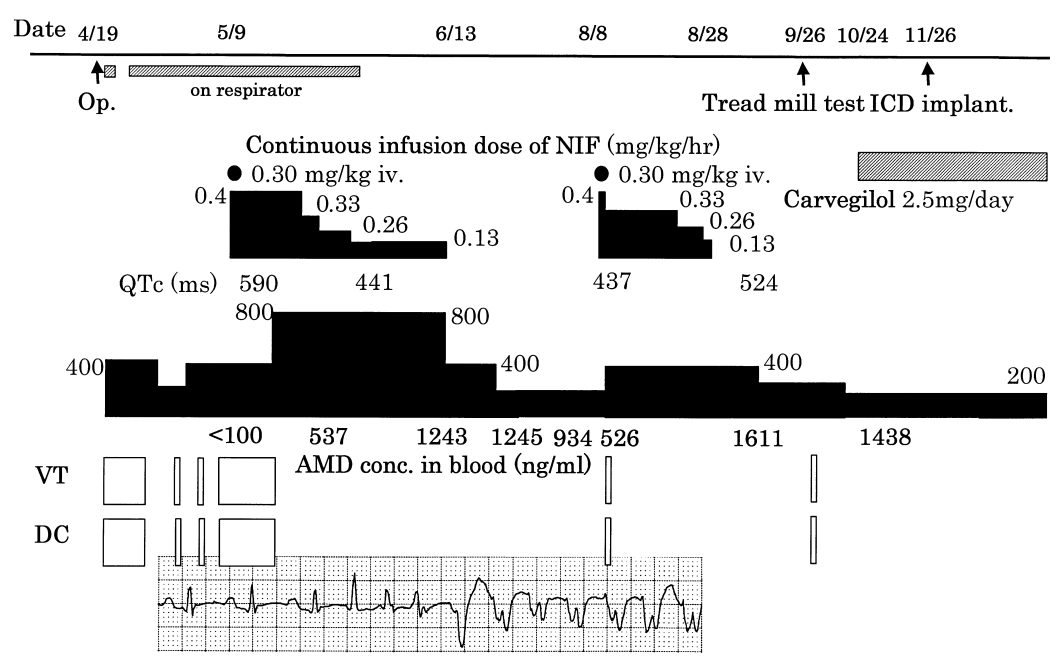

Figure. Postoperative clinical course. DC, Electrical cardioversion. 
suture was passed above the border between the scarred and normal tissue, the diameter of the neck of the contracting ventricle was reduced from $60 \mathrm{~mm} \times 50$ $\mathrm{mm}$ to $30 \mathrm{~mm} \times 25 \mathrm{~mm}$. A Meadox Hemashield patch was fixed with 3-0 Prolene continuous suture inside the LV cavity at the transitional zone marked by the purse string suture.

Despite stable postoperative circulatory status and normal serum electrolytes (potassium and magnesium) and acid-base status, the patient experienced acute onset of sustained VT at postoperative hour 4. Sinus rhythm was reestablished via electrical cardioversion. Incessant VT was subsequently induced by sympathetic stimulus, and repeated electrical cardioversion was required despite the addition of class I antiarrhythmic agents. A sedative was administered intravenously, and amiodarone (AMD, $400 \mathrm{mg} /$ day) was administered via a gastric tube. However, therapeutic AMD concentrations were not achieved, and the patient continued to experience recurrent incessant VT.

NIF was administered intravenously with a loading dose $(0.3 \mathrm{mg} / \mathrm{kg} / 5 \mathrm{~min})$ followed by an infusion $(0.4 \mathrm{mg} / \mathrm{kg} / \mathrm{hr}$ ) on May 9 (postoperative day 20). Within a few days, the patient no longer experienced recurrent VT. As the serum AMD concentrations increased, the infusion rate of the NIF was decreased, while simultaneously confirming that the goal corrected QT interval (QTc) was being maintained at less than $550 \mathrm{~ms}$. The NIF infusion was discontinued on postoperative day 55 (a duration of 35 days). The patient's hemodynamics were stable throughout the period of NIF infusion, and postoperative LV size and function improved significantly (LVEDVI $105 \mathrm{~mL} / \mathrm{m}^{2}$, LVESVI $60 \mathrm{~mL} / \mathrm{m}^{2}$, LVEF 43\%).

The patient experienced incessant VT during his daily activities. Incessant VT was again suppressed via NIF administration. However, treadmill testing performed during NIF administration induced VT when his heart rate exceeded 120 bpm. Carvedilol (2.5 mg/day) was added to the medication profile, but the patient developed multiple focused premature ventricular contractions with an increase in heart rate. Therefore, implantable cardioverter defibrillator (ICD) implantation was performed at 7 months after the Dor operation. The patient has since returned to his normal daily life, but continues to experience VT two or three times per month in the state of increasing heart rate that is successfully and painlessly controlled with antitachycardia pacing by ICD.

\section{DISCUSSION}

Dor, et al reported that one of the advantages of their LV reconstruction technique, endoventricular circular patch plasty, was reducing ventricular arrhythmias, ${ }^{1,2)}$ and it has been stressed that patients who benefit most from the Dor operation are those with more severe preoperative LV dysfunction, more fre- 
quent spontaneous and inducible VT, and higher end-systolic volumes. ${ }^{1)}$ However, as in the present case, patients with ischemic cardiomyopathy and severe global LV dysfunction are vulnerable to induction of recurrent sustained VT, especially in the perioperative period, as nonuniform conduction velocity in ischemic cardiomyopathy promotes formation of a re-entry circuit. Therefore, VT may still occur, even if cryoablation is performed in the border zone between the infarcted and noninfarcted areas. Furthermore, VT induction is increased incessantly in the context of sympathetic stimulation and worsening cardiac failure postoperatively.

Class I antiarrhythmic drugs are first line agents for VT but may also paradoxically induce VT and elevate the electrical cardioversion threshold during $\mathrm{VT}^{3)}$ as well as exert a negative inotropic effect. Further, class II antiarrhythmic agents are contraindicated in patients with severe LV dysfunction. In contrast, class III antiarrhythmic agents block potassium channels and prevent the development of VT more efficiently than class I agents. AMD is a representative class III agent that requires longer time periods to achieve therapeutic blood concentrations in conditions of intestinal paralysis, such as the perioperative period. NIF is another class III agent that can be administered intravenously and that exerts a positive inotropic effect. Therefore, NIF is particularly suited for treatment of VT in patients with severe LV dysfunction. ${ }^{4)}$

Class III agents increase cardiac refractory periods by lengthening the action potential duration (APD) and delaying recovery of excitability, and thus inhibit the re-entry circuit. But this prolongation of APD becomes less marked with an increase in heart rate. Therefore, the antiarrhythmic effect of class III agents is abated as the heart rate increases. This phenomenon has been described as "reverse use dependence". ${ }^{5}$ This is well illustrated in the present case, as the patient experienced onset of VT during periods of tachycardia. At another extreme, QT prolongation and torsades de pointes may occur with the use of class III agents during periods of bradycardia. Though NIF also had this side effect, it was possible to avoid induction of torsades de pointes with NIF in the present case by maintaining the QTc at less than $550 \mathrm{~ms}$. The SWORD (Survival With Oral d-Sotalol) trial investigated the clinical utility of the d-isomer of sotalol, a pure class III agent devoid of $\beta$-blocking activity, and demonstrated that post-MI patients with a low ejection fraction $(<40 \%)$ treated with d-sotalol had a significantly lower survival rate than those given placebo. ${ }^{6)}$ This may result from the proarrythmic effect of the drug. NIF has a strong antifibrillatory effect, although this protective effect is counterbalanced by its proarrhythmic effect. In the present case, although ICD was ultimately required, the use of NIF made suppression of VT possible during the perioperative period. 
In conclusion, intravenous infusion of NIF can be expected to be an effective, safe, and quick treatment for postoperative malignant VT refractory to class I agents with severe LV dysfunction under monitoring of QTc.

\section{REFERENCES}

1. Dor V, Sabatier M, Didonato M, Montiglio F, Toso A, Maioi M. Efficacy of endoventricular patch plasty in large postinfarction akinetic scar and severe left ventricular dysfunction: comparison with a series of large dyskinetic scars. J Thorac Cardiovasc Surg 1998; 116: 50-9.

2. Dor V. Surgery for left ventricular aneurysm. Curr Opin Cardiol 1990; 5: 773-80.

3. Murakawa Y, Yamashita T, Kanese Y, Omata M. Can a class III antiarrhythmic agent improve electrical defibrillation? J Am Coll Cardiol 1997; 29: 688-92.

4. Takenaka K, Yasuda S, Miyazaki S, et al. Initial experience with Nifekalant hydrochloride (MS551), a novel class III antiarrhythmic agent, in patients with acute extensive infarction and severe ventricular dysfunction. Jpn Circ J 2001; 65: 60-2.

5. Hondeghem LM, Snyders DJ. Class III antiarrhythmic agents have a lot of potential but a long way to go: Reduced effectiveness and dangers of reverse use dependence. Circulation 1990; 81: 686-90.

6. Waldo AI, Camm AJ, deRuyter H, et al. Effect of d-sotalol on mortality in patients with left ventricular dysfunction after recent and remote myocardial infarction. Lancet 1996; 348: 7-12. 